\title{
Computed tomography-based predictive nomogram for differentiating primary progressive pulmonary tuberculosis from community-acquired pneumonia in children
}

Bei Wang ${ }^{1 \dagger}$, Min Li ${ }^{2,3+}, \mathrm{He} \mathrm{Ma}{ }^{2}$, Fangfang Han², Yan Wang ${ }^{1}$, Shunying Zhao ${ }^{4}$, Zhimin Liu', Tong Yu' ${ }^{1}$, Jie Tian ${ }^{3,5}$, Di Dong ${ }^{3,6^{*}}$ and Yun Peng ${ }^{1,3^{*}}$ (D)

\begin{abstract}
Background: To investigate the value of predictive nomogram in optimizing computed tomography (CT)-based differential diagnosis of primary progressive pulmonary tuberculosis (TB) from community-acquired pneumonia (CAP) in children.

Methods: This retrospective study included 53 patients with clinically confirmed pulmonary TB and 62 patients with CAP. Patients were grouped at random according to a 3:1 ratio (primary cohort $n=86$, validation cohort $n=$ 29). A total of 970 radiomic features were extracted from CT images and key features were screened out to build radiomic signatures using the least absolute shrinkage and selection operator algorithm. A predictive nomogram was developed based on the signatures and clinical factors, and its performance was assessed by the receiver operating characteristic curve, calibration curve, and decision curve analysis.
\end{abstract}

Results: Initially, 5 and 6 key features were selected to establish a radiomic signature from the pulmonary consolidation region (RS1) and a signature from lymph node region (RS2), respectively. A predictive nomogram was built combining RS1, RS2, and a clinical factor (duration of fever). Its classification performance (AUC $=0.971,95 \%$ confidence interval [CI]: 0.912-1) was better than the senior radiologist's clinical judgment (AUC $=0.791,95 \% \mathrm{Cl}$ : 0.636-0.946), the clinical factor (AUC $=0.832,95 \% \mathrm{Cl}: 0.677-0.987$ ), and the combination of RS1 and RS2 (AUC = $0.957,95 \%$ Cl: $0.889-1)$. The calibration curves indicated a good consistency of the nomogram. Decision curve analysis demonstrated that the nomogram was useful in clinical settings.

Conclusions: A CT-based predictive nomogram was proposed and could be conveniently used to differentiate pulmonary TB from CAP in children.

Keywords: Child, Tuberculosis, Pulmonary, Pneumonia, Radiomics, Nomogram

\footnotetext{
* Correspondence: di.dong@ia.ac.cn; ppengyun@hotmail.com

Bei Wang and Min Li are Co-first authors.

${ }^{3}$ CAS Key Laboratory of Molecular Imaging, State Key Laboratory of

Management and Control for Complex Systems, Institute of Automation,

Chinese Academy of Sciences, No.95 Zhongguancun East Road, Beijing

100190, China

'Department of Radiology, Beijing Children's Hospital, Capital Medical

University, National Center for Children's Health, No.56 Nanlishi Road, Beijing

100045, China

Full list of author information is available at the end of the article
}

(c) The Author(s). 2019 Open Access This article is distributed under the terms of the Creative Commons Attribution 4.0 International License (http://creativecommons.org/licenses/by/4.0/), which permits unrestricted use, distribution, and reproduction in any medium, provided you give appropriate credit to the original author(s) and the source, provide a link to the Creative Commons license, and indicate if changes were made. The Creative Commons Public Domain Dedication waiver (http://creativecommons.org/publicdomain/zero/1.0/) applies to the data made available in this article, unless otherwise stated. 


\section{Background}

Pulmonary tuberculosis (TB) is one of the most widespread infections throughout the world and has a high incidence in developing countries. Pulmonary TB has a high morbidity and mortality [1], mostly in HIV-infected children [2]; however, it often occurs in children without HIV in endemic areas. It is essential for children with TB to be early diagnosed and appropriately treated with anti-TB drugs.

The positivity rate for pulmonary $\mathrm{TB}$ in children is less than $50 \%[2,3]$ due to sampling challenges and its low bacterial load. The main challenge we experienced when diagnosing pulmonary $\mathrm{TB}$ in children relates to a lack of bacteriological confirmation. Currently, the diagnosis merely relies on an examination of clinical symptoms and radiological findings, which is not accurate enough. Sreeramareddy et al. [4] reported that the delayed time for TB diagnosis in China was 25-71 days. What is more, primary progressive pulmonary $\mathrm{TB}$ can present with more severe forms such as segmental or lobar consolidation (tuberculous pneumonia or caseous pneumonia) [5]. They are more common in children than in adults [6] and are accompanied with acute symptoms that are very difficult to distinguish from the respiratory infection in children. Zaro et al. [7] reported that hospitalized children with confirmed pulmonary TB presented acute/subacute symptoms, similar to the acute pneumonia in children. The nonspecific symptoms and signs of the pulmonary TB often overlap with the common pediatric pulmonary infections and especially with those of community-acquired pneumonia (CAP). The main pathogens that cause CAP in children include Streptococcus and Mycoplasma spp., of which Streptococcus spp. is the most common, accounting for $40 \%$ of CAP cases [8]. In Asian countries, $1-7 \%$ of cases presenting with CAP are rediagnosed with pulmonary TB [9]. Therefore, it is essential to develop an effective tool for early differentiating pulmonary TB from CAP in children.

Radiomics is a novel tool adopting advanced image analysis algorithms that employ a large number of quantitative image features [10]. The integration of these features may generate powerful models to assist the disease diagnosis and prognosis [11, 12]. A number of articles have reported applications of radiomics in tumors, but few studies have reported on such applications in nonneoplastic disease.

In this study, we aimed to develop a computed tomography $(\mathrm{CT})$-based predictive nomogram for helping distinguish primary progressive pulmonary TB from CAP in children. We would also like to investigate the value of radiomics in non-neoplastic diseases.

\section{Methods}

This retrospective study was approved by the Ethics Committees of Beijing Children's Hospital for using the data, and patient consent was waived. The workflow of our study is shown in Fig. 1.

\section{Patients}

Records for pulmonary TB and CAP patients attending our institution from January 2011 to January 2018 were obtained. The patient recruitment procedure is shown in Fig. 2. A total of 53 patients with pulmonary TB and 62 patients with CAP satisfied the inclusion criteria (Fig. 3) and were included in the study. We used two symptoms (pulmonary consolidation and mediastinal lymph nodes) to build a final predictive nomogram. Patients were grouped at random according to a 3:1 ratio: 86 patients in the primary cohort and 29 patients in the validation cohort. There were 51 male patients and 35 female patients in the primary cohort, the mean age was $4.01 \pm$ 3.58 years, and an age range of $1-13$ years. The validation cohort included 19 male patients and 10 female patients with a mean age of $2.28 \pm 2.58$ years and an age range of $0-10$ years.

\section{CT examinations}

All patients underwent an unenhanced low-dose CT examination of the chest on a 64-slice Discover CT750HD scanner (GE Healthcare, Waukesha, WI, USA). The area of coverage extended from the thoracic inlet to the diaphragm. Following institutional guidelines of the low-dose CT scan protocol, all low-dose thoracic CT studies were performed using specified parameters (5 mm section thickness, $100 \mathrm{kVp}$ tube voltage, automatic tube current modulation technique, and a helical pitch of 1.375) to achieve an image noise index of 11$13 \mathrm{HU}$. The radiation dose for the patients was $1.67 \pm$ $0.83 \mathrm{mGy}$ in CT dose index volum (CTDIvol) and $41.54 \pm 22.78 \mathrm{mGy}^{*} \mathrm{~cm}$ in dose length product (DLP).

\section{CT image segmentation}

For CT image segmentation, we used an axial mediastinum window archived under the Picture Archiving and Communication System (PACS, Carestream, Vaughan, ON, Canada) for digital imaging without preprocessing or normalization.

CT images were exported to ITK-SNAP software (Version 2.2.0; http://www.itksnap.org) for manual segmentation. A radiologist with 10 years of experience performed manual segmentation in an axial mediastinal window of unenhanced CT image using a three-dimensional region of interest (ROI) to delineate the margins of pulmonary consolidation (ROI1) and mediastinal lymph nodes (ROI2). For each patient, we examined lymph nodes positioned behind the superior vena cava for delineation of ROI2. Segmentation was verified by a senior radiologist with 15 years of experience. 


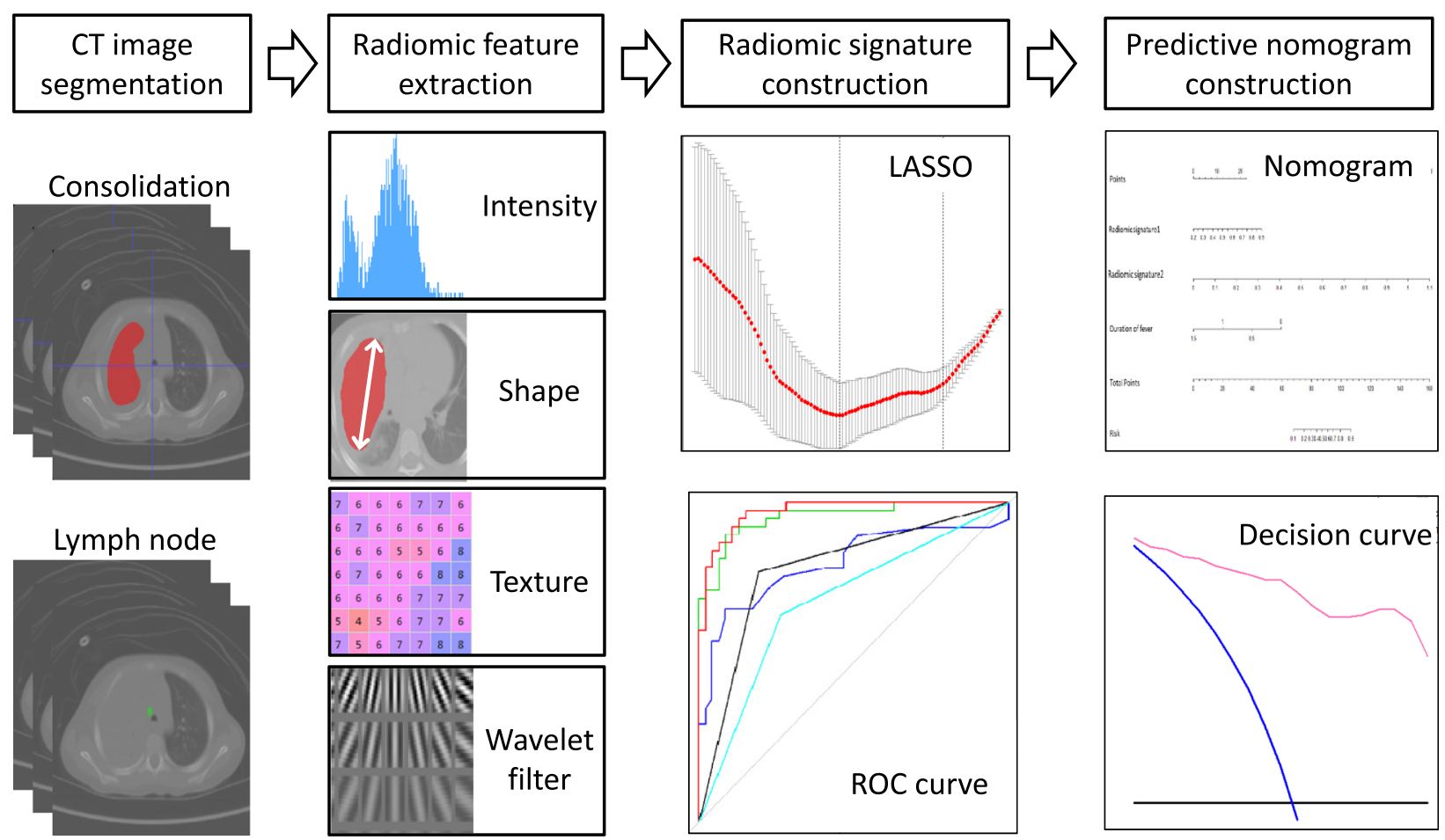

Fig. 1 The workflow of this study. The pipeline of radiomics analysis includes CT image segmentation, radiomic feature extraction, radiomic signature construction, and predictive nomogram construction

\section{Data analysis \\ Radiomic feature extraction and Radiomic signature construction}

Three-dimensional radiomic features were extracted from ROIs of the pulmonary consolidations and mediastinal lymph nodes, and each group included 485 features. These 485 features were divided into 4 categories: (a) shape and size features, (b) gray intensity features, (c) texture features, and (d) wavelet features [13]. Shape and size features reflect the phenotype of the ROIs, including the shape, area, volume, and level of compactness. Gray intensity features show differences in gray histograms and gray distributions of the ROIs. Texture features reveal the regularity of voxel relationships within the ROIs. Wavelet features reflect the transformation of gray intensity of texture features. The feature extraction method is described in detail in Additional file 1 (Appendix A1: CT feature extraction). Feature extraction was executed using MATLAB software (version 2014a; Mathworks, Natick, MA). The least absolute shrinkage and selection operator (LASSO) method is suitable for dimensionality reduction of high-dimensional data and is often used to extract the most useful features in previous studies [14].

We used the LASSO method to select key features from the radiomic features on primary cohort and built two radiomic signatures (RS1 and RS2) from ROI1 and
ROI2, respectively. Then we validated the performances of the two signatures on the validation cohort. We also constructed a radiomic model by combining the two radiomic signatures.

\section{Establishment of the predictive nomogram}

Univariate analysis was used to select significant clinical factors with $p$-values $<0.05$. Then, linear support vector machine (SVM) was used to build a predictive nomogram based on the radiomic signatures and significant clinical factors on the primary cohort. The SVM method is a generalized linear classifier for the binary classification of data in supervised learning that is widely used for pattern recognition purposes (e.g., face recognition and text categorization). In this study, The SVM is modeled using a linear kernel [15].

\section{Performance evaluation of predictive nomogram}

The performance of the predictive nomogram was evaluated on both the primary and validation cohorts. The receiver operating characteristic (ROC) curve was plotted to validate the classification ability, and calibration curves along with Hosmer-Lemeshow tests were performed to assess the goodness-of-fit of the nomogram.

For comparison, a senior radiologist (Y.W. with 6 years of experience) and a junior radiologist (T.Y. with 15 


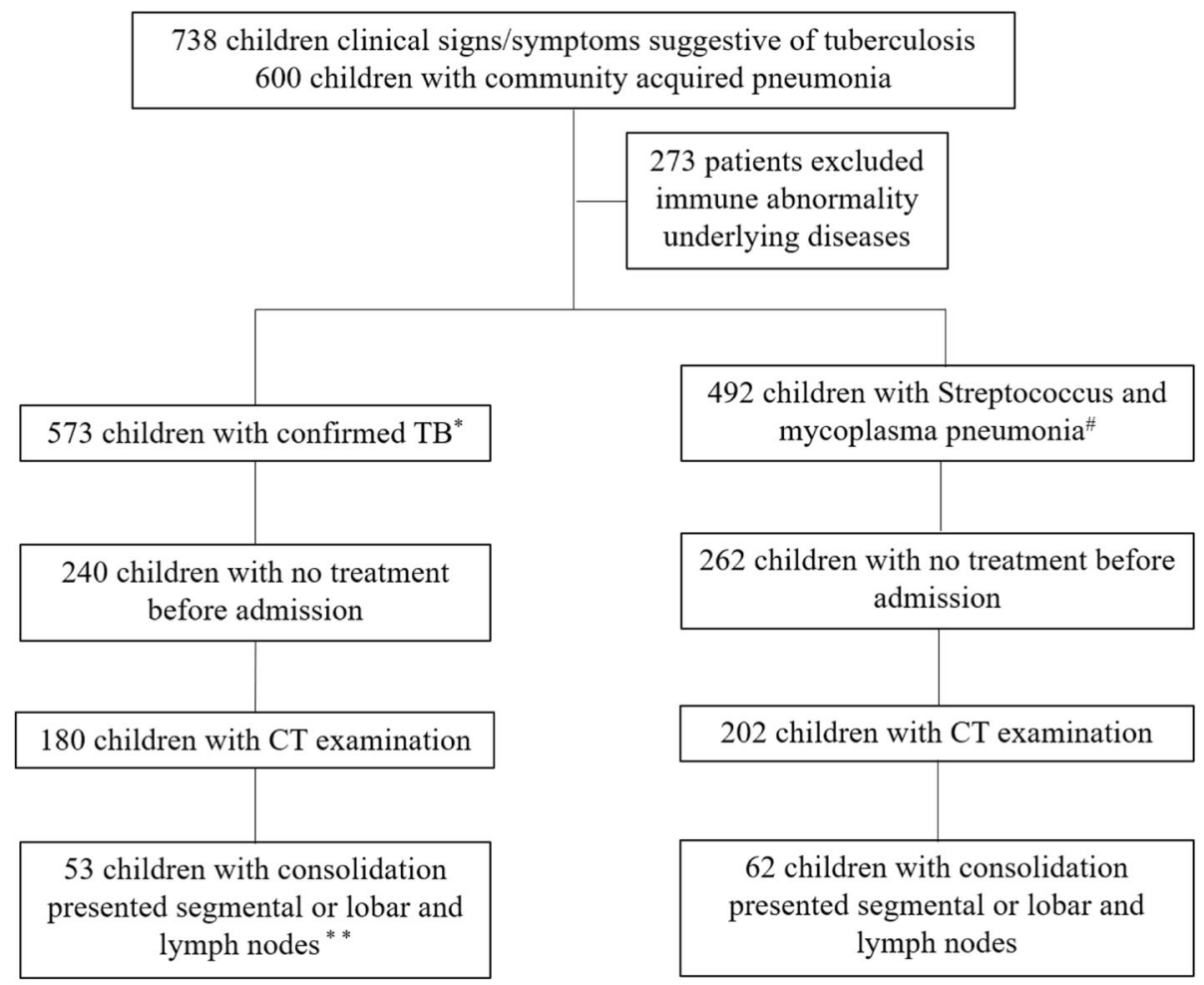

Fig. 2 Patient recruitment in this study. Note: Confirmed TB* refers to Mycobacterium tuberculosis to be confirmed (culture or Xpert MTB/RIF assay) from at least one respiratory specimen (e.g., sputum, nasopharyngeal/gastric aspirate, and pleural fluid). Lymph nodes** refers to uniformity with no calcification and necrosis in the lymph nodes. Streptococcus and mycoplasma pneumonia ${ }^{\#}$ are diagnosed via the detection of Streptococcus in pleural effusion or blood culture and positive lgM antibodies against Mycoplasma in the serum, respectively
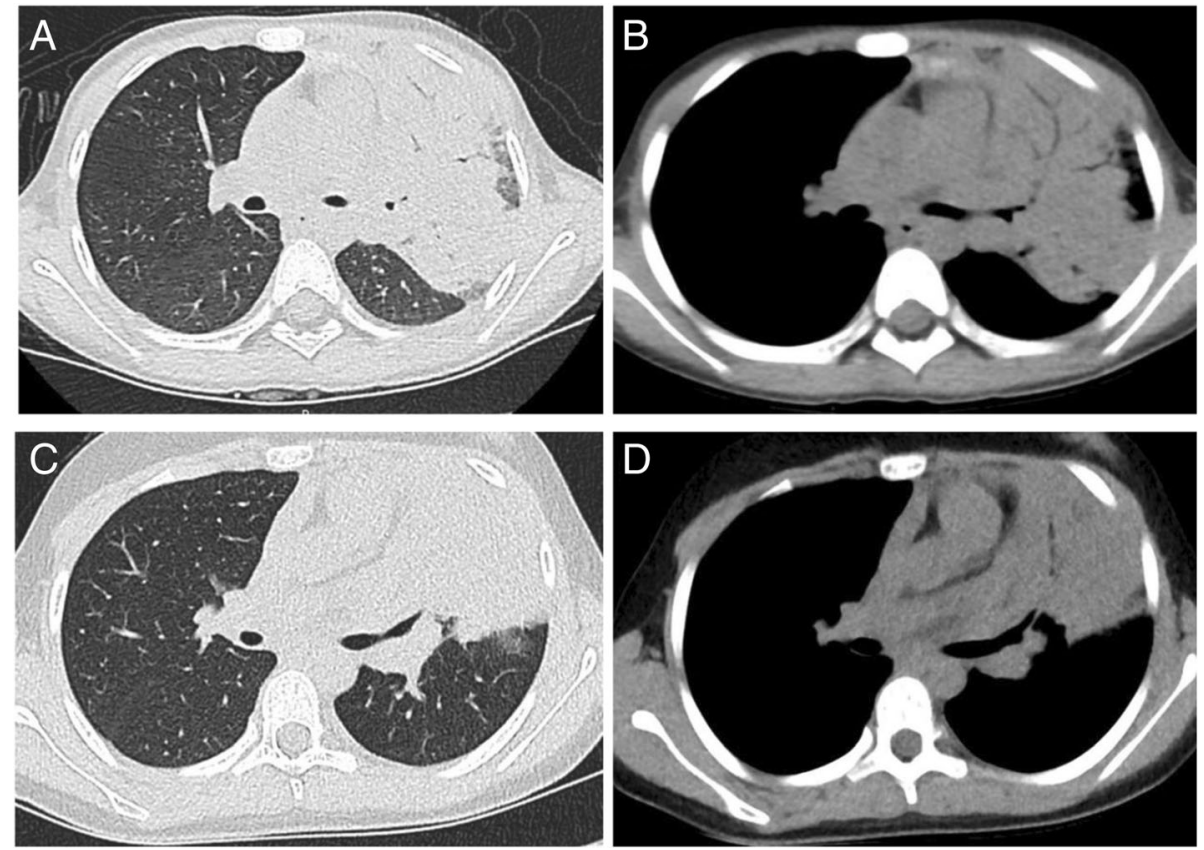

Fig. 3 Examples of CT images from two patients with tuberculosis and pneumonia. (a) and (b) show the lung window and mediastinal window of the axial CT image of a 7-year-old girl with pulmonary TB in the left upper lobe. (c) and (d) show the lung window and mediastinal window of the axial CT image of a 10-year-old girl with CAP in the left upper lobe 
Table 1 Characteristics of patients in the primary and validation cohorts

\begin{tabular}{|c|c|c|c|c|c|c|}
\hline \multirow[t]{2}{*}{ Characteristic } & \multicolumn{2}{|l|}{ Primary cohort } & \multirow[b]{2}{*}{$p$ value } & \multicolumn{2}{|l|}{ Validation cohort } & \multirow[b]{2}{*}{$p$-value } \\
\hline & CAP & Pulmonary TB & & CAP & Pulmonary TB & \\
\hline Gender, No. (\%) & & & 0.151 & & & $0.047^{*}$ \\
\hline Male & $25(54.35)$ & $26(65.00)$ & & $8(50.00)$ & $11(84.62)$ & \\
\hline Female & $21(45.65)$ & $14(35.00)$ & & $8(50.00)$ & $2(15.38)$ & \\
\hline Age, mean $\pm S D$, years & $4.51 \pm 3.44$ & $3.41 \pm 3.66$ & 0.181 & $2.69 \pm 3.10$ & $1.77 \pm 1.62$ & 0.332 \\
\hline Hemoptysis, No. (\%) & & & 0.083 & & & - \\
\hline+ & $0(0.0)$ & $3(7.50)$ & & $0(0.00)$ & $0(0.00)$ & \\
\hline- & $46(100.0)$ & 37 (92.50) & & $16(100.00)$ & $13(100.00)$ & \\
\hline Cough, No. (\%) & & & 0.324 & & & 0.82 \\
\hline- & $2(4.35)$ & $4(10.00)$ & & $0(0.00)$ & $3(23.08)$ & \\
\hline+ & $44(95.65)$ & $36(90.00)$ & & $16(100.00)$ & $10(76.92)$ & \\
\hline fever, No. (\%) & & & 0.143 & & & - \\
\hline- & $1(2.17)$ & $4(10.00)$ & & $9(56.25)$ & $5(38.46)$ & \\
\hline+ & $45(97.83)$ & $36(90.00)$ & & $7(43.75)$ & $8(61.54)$ & \\
\hline Expectoration, No. (\%) & & & 0.129 & & & 0.358 \\
\hline- & $20(43.48)$ & $24(60.00)$ & & $6(37.50)$ & $6(46.15)$ & \\
\hline+ & $26(56.52)$ & $16(40.00)$ & & $10(62.50)$ & $7(53.85)$ & \\
\hline duration of fever (days) & & & $<0.001^{* * *}$ & & & $<0.001$ \\
\hline$<10$ & $43(93.48)$ & $15(37.50)$ & & $15(93.75)$ & $3(23.08)$ & \\
\hline$\geq 10$ & $3(6.52)$ & $25(62.50)$ & & $1(6.25)$ & $10(76.92)$ & \\
\hline WBC (*10^9/L), No. (\%) & & & 0.247 & & & 0.264 \\
\hline Median(IQR) & $8.42(6.70-10.16)$ & $8.40(7.10-11.41)$ & & $14.98(14.18-17.26)$ & 16.89 (16.30-19.77) & \\
\hline Normal & $27(58.70)$ & $20(50.00)$ & & $5(31.25)$ & $5(38.46)$ & \\
\hline Abnormal & $19(41.30)$ & $20(50.00)$ & & $11(68.75)$ & $8(61.54)$ & \\
\hline CRP (mg/L), No. (\%) & & & 0.365 & & & 0.381 \\
\hline Median (IQR) & $23.30(11.53-56.25)$ & $15.70(9.44-33.88)$ & & $28.25(16.00-71.75)$ & $29.00(14.00-53.60)$ & \\
\hline Normal & $7(15.22)$ & $8(20.00)$ & & $1(6.25)$ & $3(23.08)$ & \\
\hline Abnormal & $39(84.78)$ & $32(80.00)$ & & $15(93.75)$ & $10(76.92)$ & \\
\hline Radiomic signature 1 median & 0.53 (0.32 to 0.86$)$ & & $<0.001^{* * *}$ & 0.53 (0.33 to 0.74$)$ & & $0.018^{*}$ \\
\hline Radiomic signature2 median & 0.54 (0.12 to 0.98$)$ & & $<0.001^{* * *}$ & $0.54(0.20$ to 1.26$)$ & & $<0.001^{* * *}$ \\
\hline
\end{tabular}

NOTE. The $p$-value was derived from univariable association analyses of each characteristic and of the two diseases. ${ }^{*}$ denotes $p$-value $<0.05 ;{ }^{*}$ denotes $p$-value $<$ $0.01 ;{ }^{* * *}$ denotes $p$-value $<0.001$

Abbreviations: WBC white blood cell, CRP C-reaction protein

years of experience) independently reviewed the CT images with clinical information and reached final diagnosis. The clinical judgments by radiologists were also evaluated using the area under the ROC curve (AUC) value.

\section{Clinical use}

By quantifying the net benefit to the patient under different threshold probabilities, the clinical application value of the nomogram was determined through the decision curve analysis.

\section{Statistical analysis}

Statistical analysis was performed using R software (version3.3.4; http://www.Rproject.org). A two-sided $p$-value $<0.05$ was used to indicate statistical significance. The glmnet package was used to implement the LASSO regression analysis. The pROC package was used to construct the ROC curve.

Univariate analysis was used to estimate the relationship between each patient's clinical factors and the identification of the two diseases. Independent $t$-tests or Mann-Whitney U continuous variable tests were used to assess the differences in patient variables across the 
Table 2 Radiomic feature selection results based on LASSO

\begin{tabular}{|c|c|c|c|c|}
\hline Region & Features & Group & Filters & $p$-value \\
\hline & X7_fos_maximum & Intensity & $X_{H H L}$ & $<0.001^{* * *}$ \\
\hline & X0_GLCM_maximum_probability & GLCM & $X_{L L L}$ & $0.008^{* *}$ \\
\hline \multirow[t]{3}{*}{ consolidation } & X6_GLCM_IMC1 & GLCM & $X_{H L H}$ & $<0.001^{* * *}$ \\
\hline & X1_GLRLM_LRLGLE & GLRLM & $X_{L L H}$ & $0.002^{* *}$ \\
\hline & X1_GLRLM_LRE & GLRLM & $X_{L L H}$ & $0.002^{* *}$ \\
\hline \multirow[t]{6}{*}{ lymph node } & Max3D & Shape & NA & $<0.001^{* * *}$ \\
\hline & Sph_dis & Shape & NA & $<0.001^{* * *}$ \\
\hline & Compactness 1 & Shape & NA & $<0.001^{* * *}$ \\
\hline & Surface_to_volume_ratio & Shape & NA & $<0.001^{* * *}$ \\
\hline & X2_fos_minimum & Intensity & $X_{H L H}$ & $<0.001^{* * *}$ \\
\hline & XO_GLRLM_LRHGLE & GLRLM & $X_{H H L}$ & $<0.001^{* * *}$ \\
\hline
\end{tabular}

NOTE. ${ }^{*}$ denotes $p$-value $<0.05 ;{ }^{* *}$ denotes $p$-value $<0.01 ;{ }^{* * *}$ denotes $p$-value $<0.001$

groups, and Fisher's exact tests or chi-square tests were applied for categorical variables.

All methods were assessed using the ROC curves and compared by the AUCs along with the DeLong test. The point corresponding to the maximal Youden's index on the ROC curve of the primary cohort was used as the optimal threshold value and was also applied to the validation cohort. Sensitivity and specificity were calculated to assess the model performance.

Parts of the codes used in the study are shown in the Additional file 1: Appendix code.

\section{Results}

\section{Clinical factors}

Clinical factors (gender, age, hemoptysis, cough, fever, expectoration, white blood cell (WBC) count, and C-creative protein $(\mathrm{CRP})$ ) were found not significantly different between the two diseases, while the duration of fever was found significantly associated with the two diseases according to the univariate analysis $(p<0.05$, Table 1$)$ on the primary and validation cohorts. The probability of a patient suffering from pulmonary TB and CAP was not significantly different between the two groups $(p=0.962)$

\section{Construction of the Radiomic signature}

A total of 970 radiomic features were extracted from the CT images (485 features from pulmonary consolidation regions and 485 from lymph node regions). The LASSO regression graph of these radiomic features is shown in Additional file 1 (Appendix Figure S1: The process of radiomic features selection using LASSO regression for RS1 and RS2) where key features for building the radiomic signatures are presented. Eleven key features highly related with the identification of the two diseases in the primary cohort were selected $(p<0.05$, Table 2$)$. Shape features such as "Surface_to_volume_ratio" calculates the surface area to volume ratio of the ROI, which describes the sphericity of the lesion, with lower values indicating a more compact spherical shape. First-order statistical feature "fos_maximum" and "fos_minimum" calculates the maximum and minimum grayscale intensities of the image, and describes the brightest and darkest image information of the image. Texture features such as "LRE" are calculated by the distribution of the image grayscale run matrix. The larger value of the LRE, the coarser of the texture in the ROI. Five features were extracted from the consolidation region (ROI1) and merged as a radiomic signature RS1. The other 6 features were extracted from the lymph node region (ROI2) and merged as a radiomic signature RS2. Significant differences of the radiomic signatures between pulmonary $\mathrm{TB}$ and CAP groups were found in both the primary cohort and the validation cohorts $(p<0.01$, Table 1$)$. A radiomic model was also built merging RS1 and RS2. The calculation formula of RS1 and RS2 are shown in Additional file 1 (Appendix A2: Radiomic signatures calculation formula).

\section{Predictive nomogram construction and validation}

Two radiomic signatures (RS1 and RS2) and the duration of fever were identified as independent predictors of pulmonary TB and CAP. As shown in Fig. 4(a), a predictive nomogram was built by combining RS1, RS2, and duration of fever. The performances of RS1, RS2, radiomic model, clinical factor, and predictive nomogram are shown in Table 3 . The predictive nomogram had the best differentiation ability of the two diseases with an AUC of 0.977 (95\% CI, 0.953-1) on the primary cohort and an AUC of 0.971 (95\% CI, 0.912-1) on the validation cohort, as shown in Fig. 5(a, b). In the primary cohort, the AUC value diagnosed by the senior radiologist was 0.799 (95\% CI, 0.716-0.884), with an accuracy of 0.802 (95\% CI, 0.711-0.872); and the AUC value diagnosed by the junior radiologist was 0.700 (95\% CI, 0.602-0.797), with an accuracy of 0.698 (95\% CI, 0.608- 


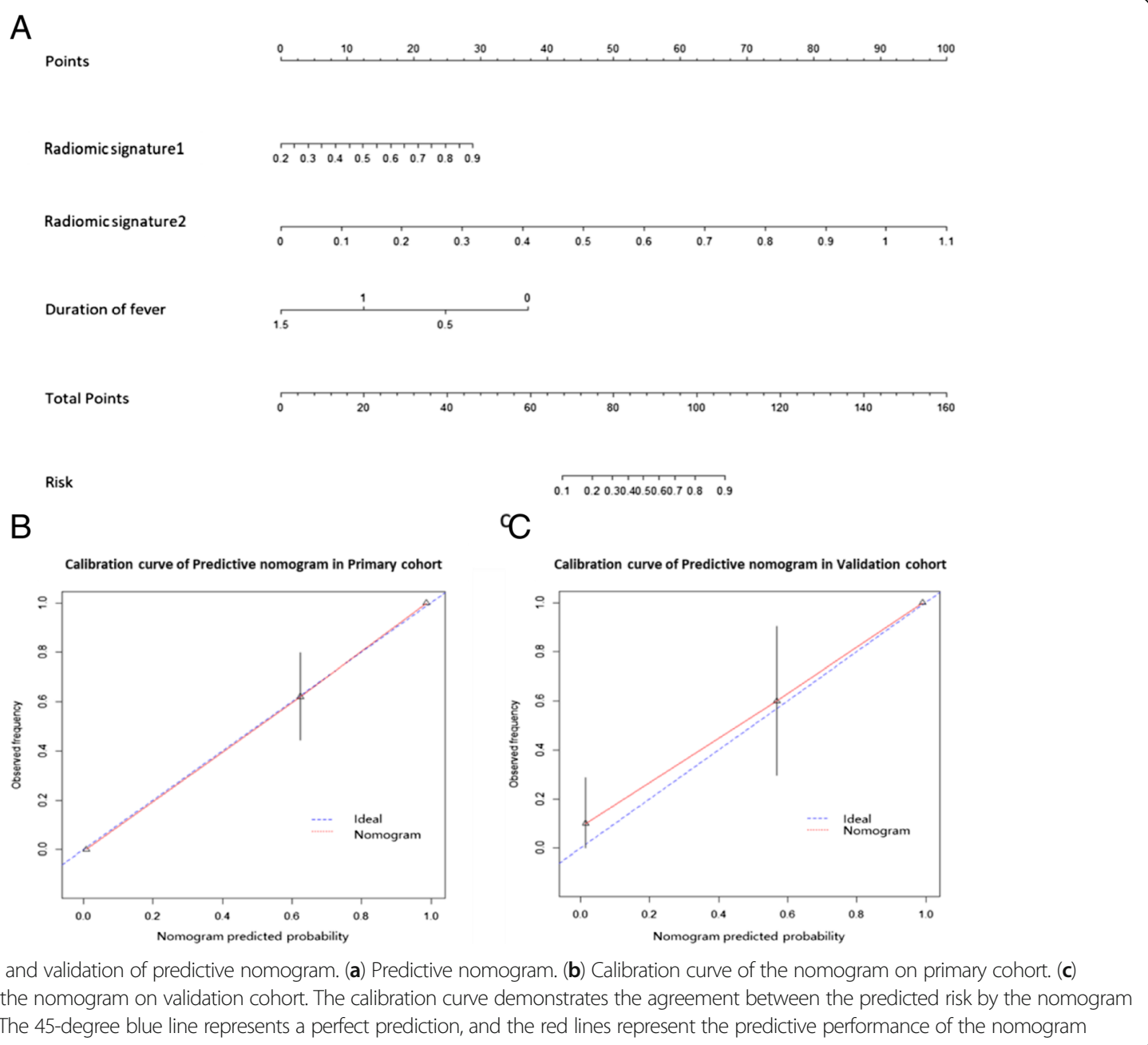

0.790). In the validation cohort, the AUC value diagnosed by the senior radiologist was 0.791 (95\% CI, 0.636-0.946), with an accuracy of 0.793 (95\% CI, 0.6030.920); and the AUC value diagnosed by the junior radiologist was 0.721 (95\% CI, 0.551-0.892), with an accuracy of 0.724 (95\% CI, 0.528-0.873).

The calibration curves of the nomogram in Fig. 4(b, c) showed that the predictions agreed well with the observations. The Hosmer-Lemeshow test results were not significant $(p>0.05)$, indicating no deviation from a perfect fit.

\section{Clinical use}

Figure 6 illustrates the decision curve analysis of the predictive nomogram. The threshold probability level is the point at which the expected benefit of treatment is equal to the expected benefit of avoiding treatment. Our nomogram showed better treatment benefit than both "treating all patients as CAP" and "treating all patients as pulmonary TB" strategies.

\section{Discussion}

To our knowledge, no previous study has analyzed cases of primary progressive pulmonary TB and CAP using radiomics. In our study, the predictive nomogram was found to be more effective than radiomic signatures of pulmonary consolidation/lymph nodes or clinical factors alone. Moreover, the diagnostic accuracy of the predictive nomogram was better than the radiologists' subjective judgments. The predictive nomogram was based on routine $\mathrm{CT}$ scan and clinical factor, which was easy to use in the clinical practice. Therefore, this predictive nomogram may serve as a potential tool for distinguishing these two major pulmonary diseases in children.

Nambu [16] demonstrated that pulmonary TB could manifest as CAP. In the early diagnostic stage, it is difficult to distinguish the pulmonary TB from CAP. In our study, only roughly $75.47 \%$ of pulmonary $\mathrm{TB}$ cases were correctly diagnosed in the entire cohort by senior radiologist, echoing the results of previous studies conducted in Iran [17], Hong Kong [18], and 


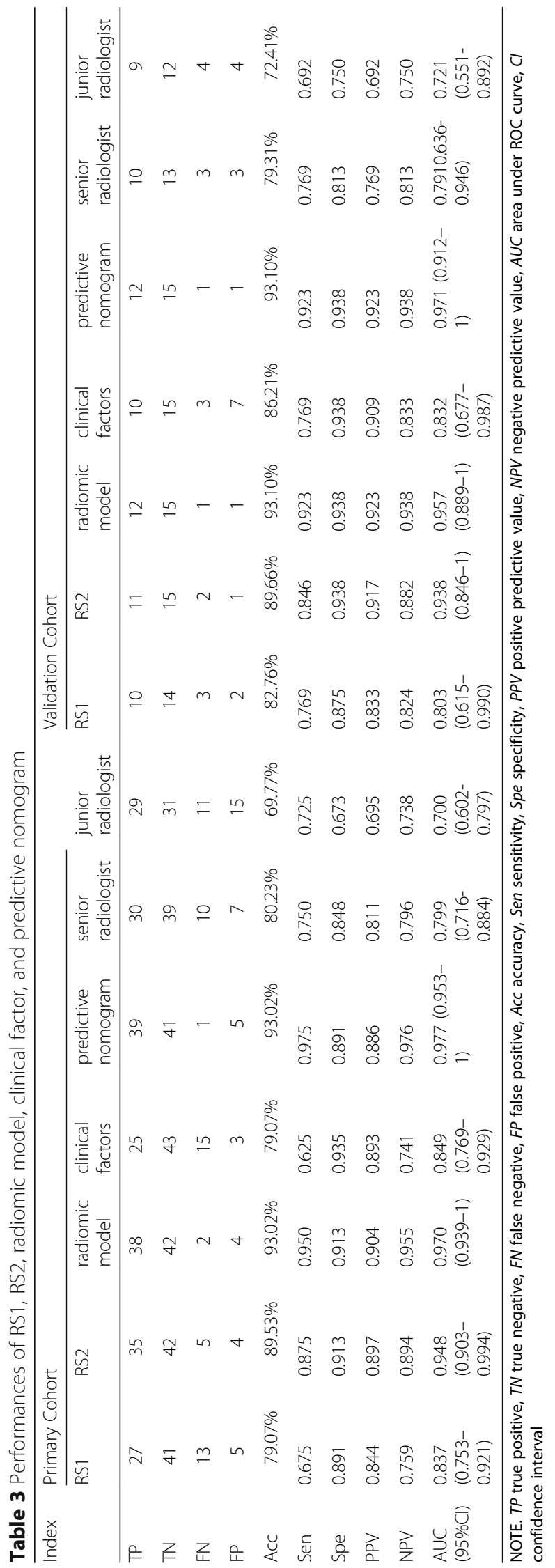



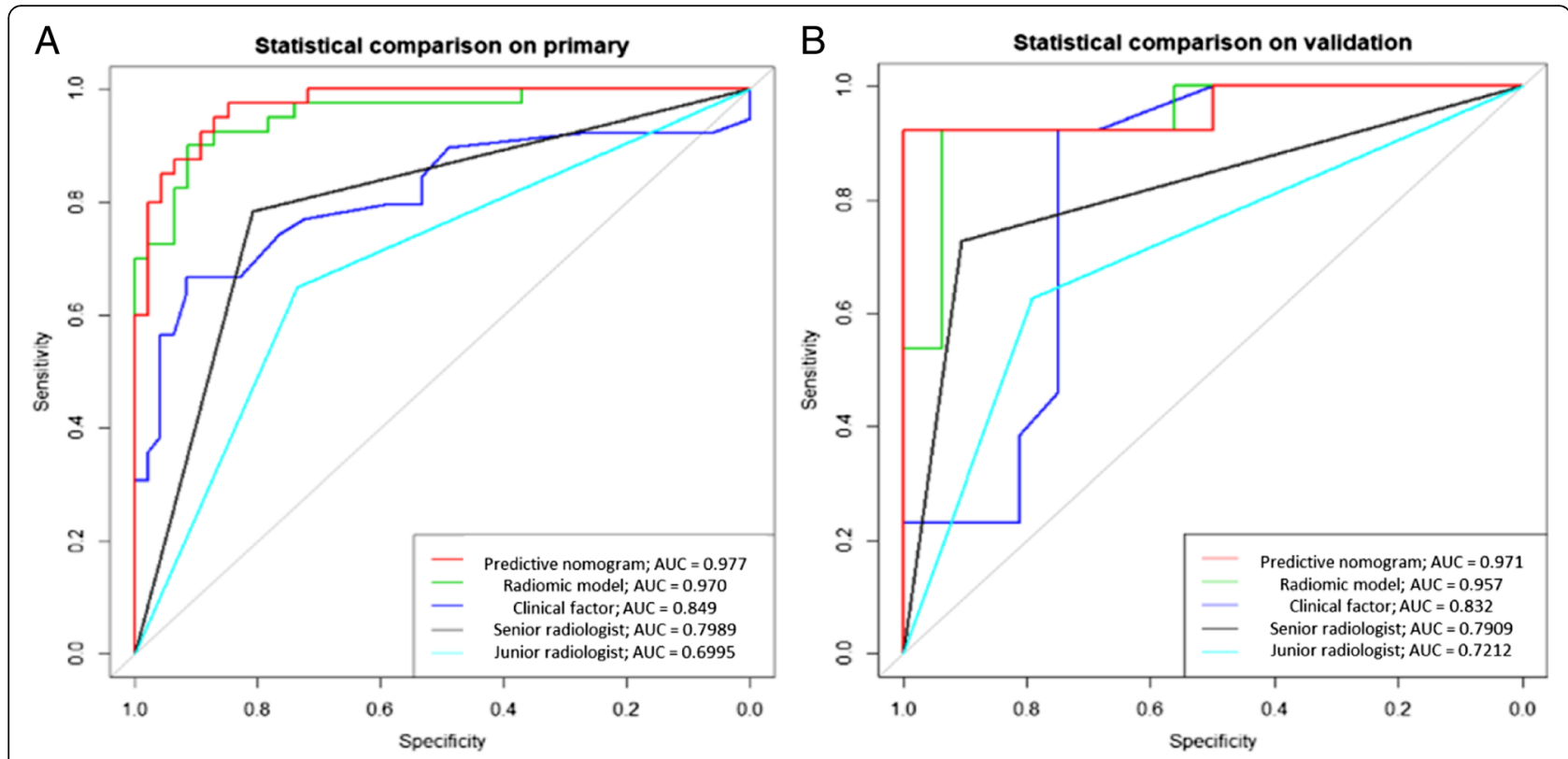

Fig. 5 Receiver operating characteristic curve analysis of the models and radiologists' diagnoses on the primary cohort (a) and validation cohort (b). The red, green, dark, black, and light blue lines denote the results of radiomic nomogram, radiomic model, clinical factors, a senior radiologist, and a junior radiologist, respectively

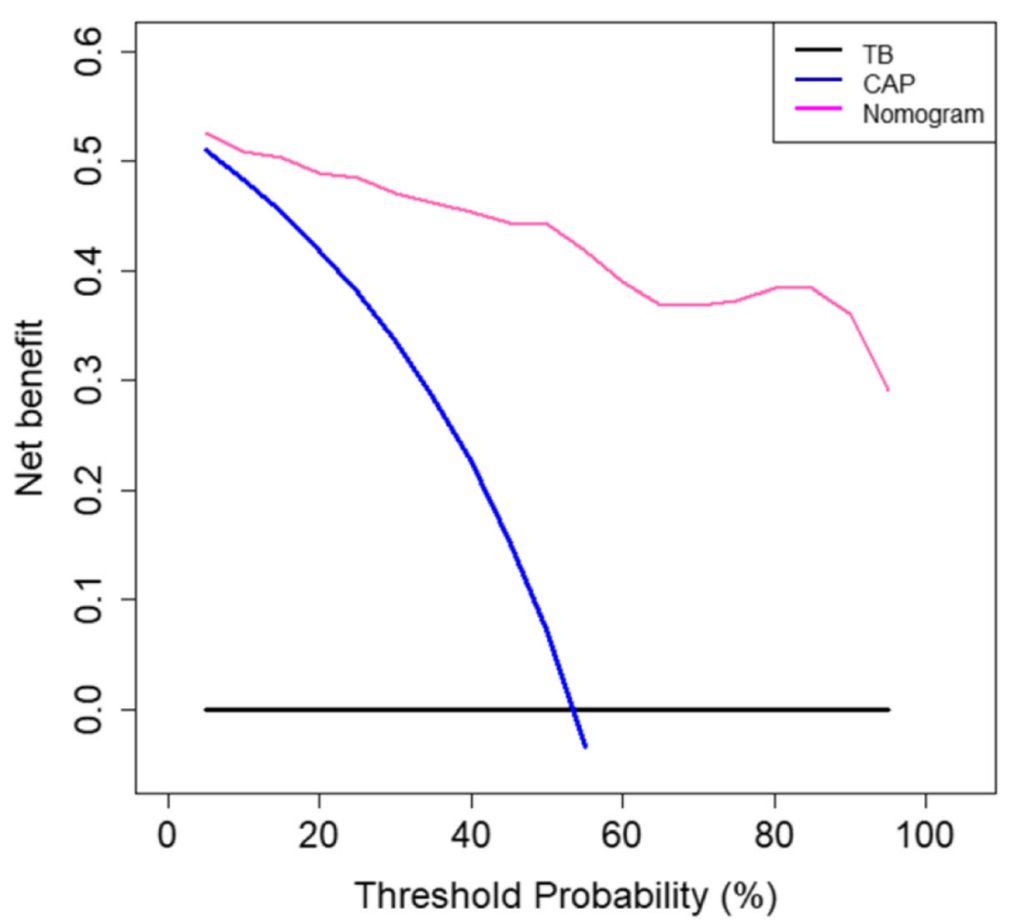

Fig. 6 Decision curve analysis of the predictive nomogram. The $x$-axis and $y$-axis represent the threshold probability value and the net benefit, respectively. The red, blue, and black lines represent the treatment benefits using the nomogram, treating all patients as CAP, and treating all patients as pulmonary TB 
Singapore [19]. Typical CT manifestations of pulmonary TB [20] include centrilobular nodules, cavities, lymph nodes containing calcification densities, and caseous necrosis. However, in the present study, all children with pulmonary TB only exhibited segmental or lobar pulmonary consolidation and lymph nodes on unenhanced CT images without any typical CT features of pulmonary TB. These CT manifestations are similar to those of common CAP with lung lobar distribution. It is thus highly challenging to differentiate the two diseases via visual assessment. Moreover, the CRP values of patients with pulmonary TB were found significantly higher than normal in this study; these CRP values were similarly elevated in patients with CAP [21]. The radiologists were also unable to get more useful information through laboratory examinations. Thus, the diagnostic rate achieved by the radiologists was lower than that achieved by the predictive nomogram.

The field of radiomics has demonstrated its potential capacity to capture useful information using machine learning methods and to enhance the accuracy of clinical differential diagnosis. In our study, 970 candidate features were extracted from $\mathrm{CT}$ images and were reduced to only 11 potential predictors by using a LASSO regression model to develop the radiomic signatures. The 11 radiomic features derived from pulmonary consolidation and lymph nodes were divided into four types (shape, texture, gray intensity features, and wavelet features) and varied significantly between cases of primary progressive pulmonary TB and CAP. Lymph nodes are complex in structure and contain microscopic textural features from unenhanced $\mathrm{CT}$ images but imperceptible to the naked eye. In this study, Max3D, Sph_dis, Compactness1, and Surface_to_volume_ratio parameters were obtained from shape features, which described the overall shapes and sizes of lymph nodes or other properties of lymph node outlines. These features were all associated with the diagnosis of the two diseases.

In our study, texture (GLCM and GLRLM) and gray intensity features extracted from the pulmonary consolidation and lymph nodes were significant radiomic features of the two diseases, but an exact clinical explanation for this remains undetermined. According to a previous study, the features often capture textural variations to quantify the spatial relationships of voxels within an image. For example, they can quantify voxels when they present similar values (e.g., related to necrosis) or spatial variations (e.g., related to intratumor heterogeneity) [22]. In our study, the Long Run Emphasis (LRE) of the texture feature was significantly greater in cases of pulmonary $\mathrm{TB}$, presumably reflecting the coarser structural textures of pulmonary consolidation when compared with lesions of CAP. In clinical cancer research, the texture features were proven to reflect the image heterogeneity of the tumor [23, 24], and thus indicated the genetic heterogeneity and invasiveness of the tumor. We speculate that the image heterogeneity of pulmonary consolidation and lymph nodes varies between pulmonary $\mathrm{TB}$ and CAP.

However, this study still presents some limitations. As a retrospective study, most cases of CAP were not subjected to enhanced CT examination. Therefore, the manual delineation of lymph node ROIs was subject to the experience of the radiologists, which may have affected the accuracy of the results. Given the patients' strict inclusion criteria, the sample size was small, which may have affected the model's reliability, and future studies should utilize larger sample size. In addition, we only examined cases involving pulmonary consolidation and lymph nodes. Other common pulmonary TB, for example with "tree bud" sign [25], should be further studied.

\section{Conclusion}

In conclusion, we proposed a CT-based predictive nomogram to differentiate primary progressive pulmonary $\mathrm{TB}$ and CAP. The CT-based predictive nomogram could serve as a new differential diagnostic tool for pulmonary infection diseases for pediatricians and radiologists.

\section{Additional file}

Additional file 1: (DOCX $259 \mathrm{~kb})$

\section{Abbreviations}

CAP: Community-acquired pneumonia; CT: Computed tomography; LASSO: Least absolute shrinkage and selection operator; LRE: Long Run Emphaisi; ROC: Receiver operating characteristic; ROI: Region of interest; RS1: Radiomic signature from pulmonary consolidation; RS2: Radiomic signature from lymph node; SVM: Support Vector Machine; TB: Pulmonary tuberculosis

\section{Acknowledgments}

Not applicable.

\section{Authors' contributions \\ The study concepts and design are completed by YP, BW, DD, and ML; the data acquisition is completed by BW, YW, TY, ZL, and SZ; the statisical analysis, algorithm design, and verification are completed by $\mathrm{ML}, \mathrm{FH}, \mathrm{HM}$, and DD; the manuscript preparation, editing, and review are completed by YP, BW, DD, ML, and JT. All authors read and approved the final manuscript.}

\section{Funding}

This work was supported by the National Key R\&D Program of China (2017YFC1308700, 2017YFA0205200, 2017YFC1309100, 2016YFA0100900, 2016YFA0100902), National Natural Science Foundation of China (81771924, 81501616, 81227901, 81671851, 81527805), the Beijing Natural Science Foundation (L182061), the Bureau of International Cooperation of Chinese Academy of Sciences (173211KYSB20160053), the Instrument Developing Project of the Chinese Academy of Sciences (YZ201502), Open Foundation of The State Key Laboratory for Management and Control of Complex Systems (20170110), and the Youth Innovation Promotion Association CAS (2017175). The Special Fund of the Pediatric Medical Coordinated Development Center of Beijing Hospitals Authority [XTCX201814]. 


\section{Availability of data and materials}

The datasets used and/or analyzed during the current study available from the corresponding author on reasonable request.

\section{Ethics approval and consent to participate}

This retrospective study was approved by the Ethics Committees of Beijing Children's Hospital for using the data, and parent consent was waived.

\section{Consent for publication}

Not applicable.

\section{Competing interests}

The authors declare that they have no competing interests.

\section{Author details}

'Department of Radiology, Beijing Children's Hospital, Capital Medical University, National Center for Children's Health, No.56 Nanlishi Road, Beijing 100045, China. ${ }^{2}$ Sino-Dutch Biomedical and Information Engineering School, Northeastern University, No. 3-11 Wenhua Road, Shenyang, China. ${ }^{3}$ CAS Key Laboratory of Molecular Imaging, State Key Laboratory of Management and Control for Complex Systems, Institute of Automation, Chinese Academy of Sciences, No.95 Zhongguancun East Road, Beijing 100190, China. ${ }^{4}$ Department of Respiratory Medicine, Beijing Children's Hospital, National Center for Children's Health, Capital Medical University, No.56 Nanlishi Road, Beijing 100045, China. ${ }^{5}$ Beijing Advanced Innovation Center for Big Data-Based Precision Medicine, School of Medicine, Beihang University, No. 37 Xueyuan Road, Beijing 100191, China. 'University of Chinese Academy of Sciences, No.19 Yuquan Road, Beijing, China.

Received: 11 March 2019 Accepted: 24 June 2019

Published online: 08 August 2019

\section{References}

1. Zumla A, George A, Sharma V, Herbert RH, Baroness Masham of I, Oxley A, Oliver M. The WHO 2014 global tuberculosis report--further to go. Lancet Glob Health. 2015;3(1):e10-2.

2. Nicol MP, Zar HJ. New specimens and laboratory diagnostics for childhood pulmonary TB: progress and prospects. Paediatr Respir Rev. 2011;12(1):16-21.

3. Zar HJ, Connell TG, Nicol M. Diagnosis of pulmonary tuberculosis in children: new advances. Expert Rev Anti-Infect Ther. 2010;8(3):277-88.

4. Sreeramareddy CT, Panduru KV, Menten J, Van den Ende J. Time delays in diagnosis of pulmonary tuberculosis: a systematic review of literature. BMC Infect Dis. 2009;9:91.

5. Veedu PT, Bhalla AS, Vishnubhatla S, Kabra SK, Arora A, Singh D, Gupta AK Pediatric vs adult pulmonary tuberculosis: a retrospective computed tomography study. World J Clin Pediatr. 2013;2(4):70-6.

6. Mukund A, Khurana R, Bhalla AS, Gupta AK, Kabra SK. CT patterns of nodal disease in pediatric chest tuberculosis. World J Radiol. 2011;3(1):17-23.

7. Zar HJ, Workman LJ, Little F, Nicol MP. Diagnosis of pulmonary tuberculosis in children: assessment of the 2012 National Institutes of Health expert consensus criteria. Clin Infect Dis. 2015;61(Suppl 3):S173-8.

8. Oliwa JN, Karumbi JM, Marais BJ, Madhi SA, Graham SM. Tuberculosis as a cause or comorbidity of childhood pneumonia in tuberculosis-endemic areas: a systematic review. Lancet Respir Med. 2015;3(3):235-43.

9. Shen GH, Tsao TC, Kao SJ, Lee J], Chen YH, Hsieh WC, Hsu GJ, Hsu YT, Huang CT, Lau YJ, et al. Does empirical treatment of community-acquired pneumonia with fluoroquinolones delay tuberculosis treatment and result in fluoroquinolone resistance in mycobacterium tuberculosis? Controversies and solutions. Int J Antimicrob Agents. 2012:39(3):201-5.

10. Zhang B, Tian J, Dong D, Gu D, Dong Y, Zhang L, Lian Z, Liu J, Luo X, Pei $S$, et al. Radiomics features of multiparametric MRI as novel prognostic factors in advanced nasopharyngeal carcinoma. Clin Cancer Res. 2017;23(15):4259-69.

11. Dong D, Tang L, Li ZY, Fang MJ, Gao JB, Shan XH, Ying XJ, Sun YS, Fu J, Wang $X X$, et al. Development and validation of an individualized nomogram to identify occult peritoneal metastasis in patients with advanced gastric cancer. Ann Oncol. 2019;30(3):431-8.

12. Peng $H$, Dong $D$, Fang $M J$, Li L, Tang LL, Chen L, Li WF, Mao YP, Fan W, Liu $L Z$, et al. Prognostic value of deep learning PET/CT-based Radiomics: potential role for future individual induction chemotherapy in advanced nasopharyngeal carcinoma. Clin Cancer Res. 2019.
13. Zhu X, Dong D, Chen Z, Fang M, Zhang L, Song J, Yu D, Zang Y, Liu Z, Shi J, et al. Radiomic signature as a diagnostic factor for histologic subtype classification of non-small cell lung cancer. Eur Radiol. 2018;28(7):2772-8.

14. Song J, Shi J, Dong D, Fang M, Zhong W, Wang K, Wu N, Huang Y, Liu Z, Cheng $Y$, et al. A new approach to predict progression-free survival in stage IV EGFR-mutant NSCLC patients with EGFR-TKI therapy. Clin Cancer Res. 2018;24(15):3583-92

15. Team RDCJC: R Core Team R A Language and Environment for Statistical Computing 2014. 2015, 1:12-21.

16. Nambu A, Ozawa K, Kobayashi N, Tago M. Imaging of community-acquired pneumonia: roles of imaging examinations, imaging diagnosis of specific pathogens and discrimination from noninfectious diseases. World J Radiol. 2014:6(10):779-93.

17. Naderi H, Sheybani F, Erfani SS, Amiri B, Nooghabi MJ. The mask of acute bacterial pneumonia may disguise the face of tuberculosis. Electron Physician. 2017;9(3):3943-9.

18. Chan $\mathrm{CH}$, Cohen M, Pang J. A prospective study of community-acquired pneumonia in Hong Kong. Chest. 1992;101(2):442-6.

19. Hui KP, Chin NK, Chow K, Brownlee A, Yeo TC, Kumarasinghe G, Chan TB, Tan WC. Prospective study of the aetiology of adult community acquired bacterial pneumonia needing hospitalisation in Singapore. Singap Med J. 1993:34(4):329-34

20. Mehrian P, Moghaddam AM, Tavakkol E, Amini A, Moghimi M, Kabir A, Velayati A. Determining the lymphadenopathy characteristics of the mediastinum in lung CT scan of children with tuberculosis. Int $J$ Mycobacteriol. 2016:5(3):306-12.

21. Ishiguro T, Yoshii Y, Kanauchi T, Hoshi T, Takaku Y, Kagiyama N, Kurashima K, Takayanagi N. Re-evaluation of the etiology and clinical and radiological features of community-acquired lobar pneumonia in adults. J Infect Chemother. 2018;24(6):463-9.

22. Rios Velazquez E, Parmar C, Liu Y, Coroller TP, Cruz G, Stringfield O, Ye Z, Makrigiorgos M, Fennessy F, Mak RH, et al. Somatic mutations drive distinct imaging phenotypes in lung Cancer. Cancer Res. 2017:77(14):3922-30.

23. Lambin P, Leijenaar RTH, Deist TM, Peerlings J, de Jong EEC, van Timmeren J, Sanduleanu S, Larue R, Even AJG, Jochems A, et al. Radiomics: the bridge between medical imaging and personalized medicine. Nat Rev Clin Oncol. 2017:14(12):749-62.

24. Bi WL, Hosny A, Schabath MB, Giger ML, Birkbak NJ, Mehrtash A, Allison T, Arnaout $\mathrm{O}$, Abbosh $\mathrm{C}$, Dunn IF, et al. Artificial intelligence in cancer imaging: clinical challenges and applications. CA Cancer J Clin. 2019;69(2):127-57.

25. Graham SM, Cuevas LE, Jean-Philippe P, Browning R, Casenghi M, Detjen AK, Gnanashanmugam D, Hesseling AC, Kampmann B, Mandalakas A, et al. Clinical case definitions for classification of intrathoracic tuberculosis in children: an update. Clin Infect Dis. 2015:61(Suppl 3):S179-87.

\section{Publisher's Note}

Springer Nature remains neutral with regard to jurisdictional claims in published maps and institutional affiliations.

Ready to submit your research? Choose BMC and benefit from:

- fast, convenient online submission

- thorough peer review by experienced researchers in your field

- rapid publication on acceptance

- support for research data, including large and complex data types

- gold Open Access which fosters wider collaboration and increased citations

- maximum visibility for your research: over $100 \mathrm{M}$ website views per year

At BMC, research is always in progress.

Learn more biomedcentral.com/submission 